\title{
Sphenoidal mucopyocele with visual impairment: case report of a rare disease and literature review*
}

\section{Bigyan Raj Gyawali and Bibhu Pradhan}

Department of ENT-Head and Neck Surgery, Institute of Medicine, Tribhuvan University Teaching Hospital, Kathmandu, Nepal
Rhinology Online, Vol 1: 57 - 59, 2018

http://doi.org/10.4193/RHINOL/18.022

*Received for publication:

May 10,2018

Accepted: May 29, 2018

Published: June 9, 2018

\begin{abstract}
Background: The sphenoid sinus is affected in $2 \%$ of the cases presenting with a mucocele or mucopyocele of the paranasal sinuses. The close proximity of the optic nerve usually results in visual symptoms. Timely diagnosis and treatment is required to prevent permanent loss of vision.

Case: A 28 years old male presented at our center with left sided periorbital pain with progressive blurring of vision for 3 years. A mucocele of the sphenoid sinus was identified on CT and MRI scans. Endoscopic sphenoidotomy revealed a mucopyocele of the sphenoid sinus with a dehiscent roof. Marsupialization of the sac was performed. Following surgery, there was improvement of periorbital pain, however, the vision remained static without further deteoration.
\end{abstract}

Conclusion: Mucopyoceles of the sphenoid sinus require early recognition to prevent the debilitating visual symptoms. CT scan is the preferred imaging modality and endoscopic approach is the recommended approach for marsupializaion of the sac.

Key words: sphenoid, mucocele, mucopyocele, marsupialization, optic nerve

\section{Introduction}

Mucocele or mucopyocele of the nose and paranasal sinuses affecting the sphenoid sinus accounts for only $2 \%$ of the cases ${ }^{(1)}$. This entity comes with variable presentations including altered vision. The close proximity of the optic nerve accounts for the symptoms. Thus, a timely diagnosis and intervention is required in all these cases to revert, or at least halt, the debilitating visual symptoms and other complications.

\section{Case}

A twenty-eight-years old man presented to our OPD with a history of left periorbital pain for 3 years. The pain was dull in nature without any radiation, it was continuous with gradual progression, and only being relieved to some extent with analgesics. There were no known aggravating or relieving factors. It was associated with a blurred vision in the left eye with gradual onset and progression with the same duration. However, there was no history of vomiting, seizure or aura.
He didn't have any significant past medical illness such as hypertension, diabetes, tuberculosis and also didn't have any family history of migraine.

The general examination of the patient revealed no significant findings. Anterior rhinoscopy showed there was a left sided deviated nasal septum without signs of rhinitis. No mass could be visualized within the nasal cavity. Palpating the paranasal sinuses revealed no tenderness. Examination of the ears bilaterally yielded normal findings with intact tympanic membranes, positive Rinne and centralized Weber. Vestibular examination was normal. Oral cavity and neck examination were also normal. Motor and sensory functions of all 4 limbs and function of all cranial nerves were intact except for the optic nerve, which showed reduced distant vision. Nasal endoscopy revealed no abnormality except for the deviated nasal septum.

He was advised for a thorough hematological and biochemistry analysis. All reports were within normal range.

A CT scan of the nose and paranasal sinuses revealed homo- 


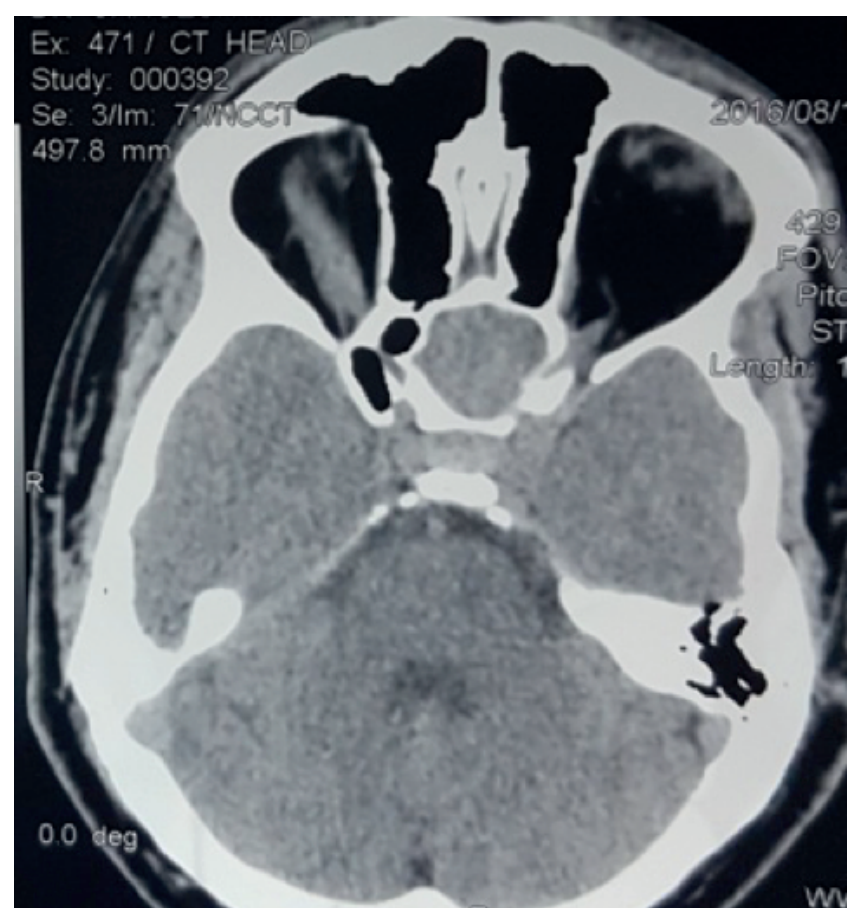

Figure 1. CT scan showing soft tissue density in sphenoid sinus.

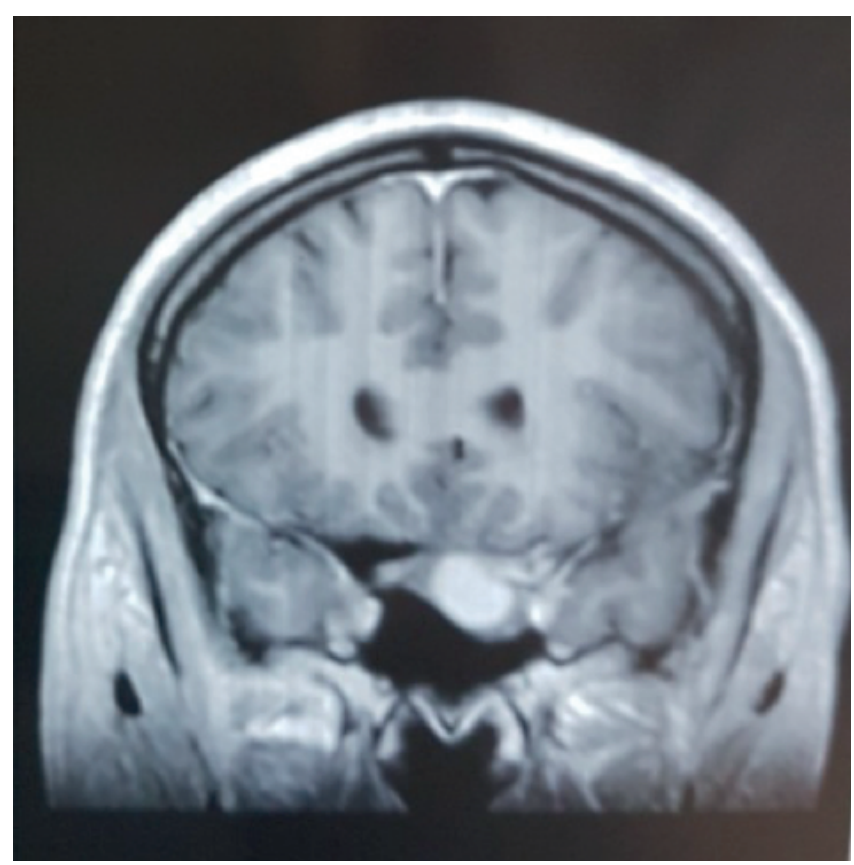

Figure 2. MRI showing soft tissue mass with moderate signal intensity in the region of sphenoid sinus. genous soft tissue density in the sphenoid sinus (Figure 1) and the MRI head showed a soft tissue mass in the region of the sphenoid sinus at the left side with moderate signal intensity in the T1 weighted image (Figure 2).

Based on the clinical findings and the imaging reports, a diagnosis of a left sphenoidal mass under evaluation was made. The patient underwent endoscopic sinus surgery. Opening the sphenoid ostium revealed a mucopyocele within the sphenoid sinus with a dehiscent roof. Endoscopic marsupialization of the mucopyocele was performed. The patient was kept under I/V Co-Amoxyclav for 3 days. The nasal pack was removed on the 2nd post-operative day and the patient was discharged on the 3rd post-operative day. On follow-up after 6 months, the patient had no symptoms of headache with static visual symptoms.

\section{Discussion}

Mucopyocele affecting the sphenoid sinus presenting with visual disturbances is a very rare presentation. Symptomatic presentations include headache, nasal discharge and rarely visual symptoms such as visual loss, decrease visual acuity and diplopia ${ }^{(2)}$. Our patient had chief complaints of left periorbital pain with blurring of vision in the left eye for 3 years. Its' location in the left side of sphenoid sinus in close proximity to the optic nerve and infective nature of the disease might account for the visual symptoms and periorbital pain.

Sinha et al. ${ }^{(3)}$ in their study reported a similar case of sphenoid mucocele presenting with complete loss of vision in the right eye. Similarly, Hill et al. ${ }^{(4)}$ in their case series reported various ocular manifestations of sphenoid mucoceles such as diplopia, reduced visual acuity, temporal hemianopia and even third and sixth nerve paresis. A retrospective analysis of 39 patients performed by Ruoppi et al. ${ }^{(5)}$ showed $28 \%$ of the patients presented with occular symptoms such as diplopia, decreased visual acuity, conjunctival irritation and photophobia and $21 \%$ of the patients presented with isolated cranial nerve palsy of second, third and fifth cranial nerve. Okuda et al. ${ }^{(6)}$ in their analysis of 44 cases of isolated sphenoid sinus lesions found that $27.0 \%$ of the cases presented with ocular symptoms that included ocular pain, visual disturbance and diplopia.

Diagnosis relies on imaging as a mucocele is usually not visualized on nasal endoscopy. CT scan and MRI are the investigation methods of choice. A mucocele appears as a homogenous, nonenhancing mass by $\mathrm{CT}$ whereas a variation in signal intensity is seen by MRI depending on its constituents.

Marsupialization of the mucocele confers curative treatment most of the times with an endoscopic approach as the preferred one. In our case, an endoscopic marsupialization was performed following sphenoidotomy. The recurrence rate is relatively lower for the endoscopic approach compared to external or combined endoscopic and external approaches ${ }^{(7)}$. Eordoh et al. ${ }^{\left({ }^{(8)}\right.}$ have described a triangular bony structure that lies anterior to the sphenopalatine foramen and is built up by the palatine, ethmoid bone and maxilla. It serves as a landmark for sphenoid sinus 
during transnasal surgery.

Prognosis of the visual symptoms following surgery depends on timing of the intervention as well as their severity. Long-standing cases with poor vision are associated with poorer prognosis compared to the cases which are intervened early, although further deterioration of vision might be halted. In our case, his periorbital pain improved following surgery, however, the vision remains static compared to the preoperative status. In the study performed by Chen et al. ${ }^{(9)}$ on 23 patients presenting with an isolated sphenoid sinus disease and visual disturbances such as reduced visual acuity and double vision, 6 had improvement following sphenoidotomy and clearance of the disease.

The recurrence rate following endoscopic approach is comparatively lower. A study performed on 59 patients with paranasal sinus mucoceles by Haiat et al. ${ }^{(10)}$ showed a recurrence rate of only $9 \%$ after endoscopic, or combined endoscopic and open approaches.

\section{Conclusion}

Due to close proximity of the optic nerve to the sphenoid sinus roof, patients with sphenoid mucocele or mucopyocele might present with visual symptoms such as diplopia, blurring of vision, reduced visual acuity, and even third, fifth and sixth nerve palsies. Such cases should be investigated thoroughly and a CT scan of the nose and paranasal sinuses is the modality of choice. All cases with visual symptoms should be intervened as early as possible to prevent further deterioration. Primary management is always surgery, preferably the endoscopic approach with drainage of mucopus and marsupialization of the sac.

\section{Consent for publication}

Written informed consent for publication of their clinical details and clinical images was obtained from the patient. A copy of the consent form is available for review by the Editor of this journal.

\section{Authorship contribution}

BG: Manuscript preparation, assistant surgeon; BP: operating surgeon.

\section{Conflict of interest}

The authors declare that they have no competing interests.

\section{References}

1. Darouassi Y, Righini CA, Reyt E. Mucoceles of the sphenoid sinus: a report of four cases and review of the literature. BENT 2005; 1: 181-5.

2. Strek P, Zagólski O, Skladzieñ J, Oles K Sphenoid sinus pyocele with intracrania extension managed under endoscopic guidance: a report of an extremely rare case. B-ENT. 2007; 3(3): 149-51.

3. Sinha B K, Adhikari P. Sphenoid sinus mucocele with blindness: a rare presentation. Nepal Med Coll J. 2008; 10: 204-206.

4. Hill C, Kumar G, Virk JS, Owa A, Kaddour H. Sphenoid mucocele: a rare cause of ocular dysfunction. QJM. 2014 Jun;107(6):463-4.

5. Ruoppi P, Seppä J, Pukkila M, Nuutinen J. Isolated Sphenoid Sinus Diseases Report of 39 Cases. Arch Otolaryngol Head Neck Surg.
2000;126(6):777-781.

6. Okuda T, Hanamure Y, Kasano F, Kashima N. Isolated sphenoid sinus lesions: a clinical analysis of 44 cases: Nippon Jibiinkoka Gakkai Kaiho 2005; 108: 835-41.

7. Lund VJ. Endoscopic management of paranasal sinus mucoceles. J Laryngol Otol. 1998; 112: 36-40.

8. Eordogh M, Grimm A, Gawish I, et al. Anatomy of the sphenopalatine artery and its implications for transnasal neurosurgery. Rhinology. 2018 Mar 1;56(1):82-88.

9. Lanlan Chen, Libin Jiang, Bentao Yang, Prem S. Subramanian. Clinical features of visual disturbances secondary to isolated sphenoid sinus inflammatory disease. BMC Ophthalmol. 2017;17:237.

10. Waizel-Haiat S, Díaz-Lara IM, Vargas-Aguayo AM, Santiago-Cordova JL. Experience in the surgical treatment of paranasal sinus mucoceles in a university hospital. Cirugía y Cirujanos (English Edition). 2017; 85: 4-1.

Dr. Bigyan Raj Gyawali

Department of ENT

Head and Neck Surgery

Institute of Medicine

Tribhuvan University

Kathmandu

Nepal

Tel: + 9779803105062

E-mail: bigyan.gyawali@gmail.com 